Instructions for authors, subscriptions and further details:

\title{
http://mcs.hipatiapress.com
}

\section{Alternative Masculinities for a Changing World}

Liviu Catalin Mara ${ }^{1}$

1) Universitat Rovira i Virgili, Spain

Date of publication: June $21^{\text {th }}, 2015$

Edition period: June 2015-October 2015

To cite this article: Mara L. (2015). Alternative masculinities for a changing world [Review of the book]. Masculinities and Social Change, 4(2), 211-212. doi: 10.17583/MCS.2015.1583

To link this article: http://doi.org/10.17583/MCS.2015.1583

\section{PLEASE SCROLL DOWN FOR ARTICLE}

The terms and conditions of use are related to the Open Journal System and to Creative Commons Attribution License (CC-BY). 


\section{Reviews (I)}

Carabí, À., \& Armengol, J. M. (Eds.). (2014). Alternative Masculinities for a Changing World. New York: Palgrave Macmillan. ISBN: 978-1-13746255-8

Este libro es una compilación de ensayos cuyo objetivo es el de posicionarse en contra de los comportamientos propios de la masculinidad hegemónica y del patriarcado para así preparar y contribuir al surgimiento de masculinidades nuevas o alternativas. Por masculinidades alternativas, los autores entienden aquellas masculinidades que: "cuestionan el comportamiento de la masculinidad hegemónica y dominante y que personifican formas más igualitarias de masculinidad. Estas masculinidades alternativas se caracterizan por no ser sexistas, ni racistas, ni homófobas, ni clasistas, etc.". (trad. propia, p. 219)

Para conseguir el objetivo principal de fomentar estas masculinidades alternativas, el libro se adentra en el estudio de comportamientos y prácticas masculinas alternativas y en la exploración de las tendencias más equitativas en cuanto a género, al mismo tiempo que presta atención a los contextos sociales y culturales en los que se desarrollan estas actitudes dentro de la literatura americana contemporánea. El resultado que se pretende es ofrecer a los hombres de todas las edades modelos de comportamiento alternativos al modelo hegemónico y dominante. De esta manera, se pretende ofrecer a los hombres la posibilidad de transformación o una construcción de masculinidad diferente para las generaciones venideras a través de la reflexión y exposición a unos procesos de socialización diferentes.

Tal vez uno de los aspectos más novedosos que aporta el libro a la vasta literatura existente en torno a las masculinidades es su estructura original en la que queda reflejada su interdisciplinariedad. El libro tiene tres partes principales. La primera parte ofrece las aportaciones teóricas a los estudios de la masculinidad desde varias disciplinas de las Ciencias Sociales, y también de las Humanidades, como Trabajo Social, Sociología, Psicología, 


\section{Mara - Alternative Masculinities [Book Review]}

Antropología y Literatura. La segunda parte consiste en la aplicación de dichos conceptos teóricos en el análisis de los modelos de masculinidad alternativos construidos en la literatura americana contemporánea. Por último, en la tercera parte del libro se reproduce una entrevista extensa sobre las masculinidades alternativas, entre los autores que impulsaron la elaboración de este libro y Victor J. Seidler, que se dedicó, entre otras, al estudio de la masculinidad.

En el recorrido que se hace en la segunda parte del libro por la literatura americana contemporánea, los autores exploran diferentes aspectos de la paternidad como constituyentes de un posible modelo alternativo; modelos de masculinidad no violentos; los modelos de masculinidad chicano/latino y los modelos de masculinidad alternativos en la población afro-americana. Para esto último analizan tres obras de Toni Morrison, Premio Pulitzer y Premio Nobel de Literatura. Por otro lado, también se exploran prácticas alternativas de género, cómo la construcción de prácticas transformadoras de la masculinidad a raíz de la crisis económica del 2008, o la reconstrucción de las masculinidades en el caso de los inmigrantes con el foco puesto en los árabes de los Estados Unidos de América.

Este libro aporta un enfoque novedoso en el estudio de las masculinidades a través del análisis de diferentes modelos de masculinidades construidos dentro de una literatura determinada, con el propósito de ampliar a nivel teórico el debate sobre los diferentes modelos de masculinidad. Tal vez hubiera sido interesante que los autores del libro plantearan como se puede llegar a contrarrestar en la práctica el modelo de la masculinidad hegemónica por las alternativas que presentan en este estudio. Sin embargo, desde el principio se avisa a los lectores que este libro "no pretende cuestionar (si fuera posible) la hegemonía masculina ni se pretende cuestionar le hegemonía de unos hombres sobre otros hombres" sino que pretende demostrar que dicha hegemonía no es universal ni inmutable, y que contiene muchas contradicciones internas a nivel global.

Liviu Catalin Mara, Universitat Rovira i Virgili liviucatalin.mara@urv.cat 